\title{
Editorial: Environmental Psychology and the Built Environment
}

\author{
Lindsay J. McCunn ${ }^{1 *}$ and Amy Kim ${ }^{2}$ \\ ${ }^{1}$ Department of Psychology, Vancouver Island University, Nanaimo, BC, Canada, ${ }^{2}$ Department of Civil and Environmental \\ Engineering, University of Washington, Seattle, WA, United States
}

Keywords: environmental psychology, sustainability, built environment, community, construction

\section{Editorial on the Research Topic}

\section{Environmental Psychology and the Built Environment}

We are pleased to offer this edited collection of articles that convey innovative outcomes and methods as a result of considering environmental psychology in the study of built settings. Although using psychological science to inform designs that optimize social interaction and environmental perception is not new, environmental psychology is still a young sub-field. Thus, we wished to curate an interdisciplinary topic to educate and assist researchers and practitioners about how human perception, attitudes, and behaviors can be applied to projects in engineering, facilities management, community planning, and beyond.

Six papers are included in this collection. Two focus on changing behaviors at work-a theme that many environmental and conservation psychologists investigate. In the paper "Changing Behavior Through Design: A Lab Fume Hood Closure Experiment," Cheek and Wells examine ways to promote behavior change for people using fume hoods in laboratories. One hood can use as much energy as three homes can in a year! Communication to users (i.e., on a sticker), along with feedback from automatized building equipment, were used to test whether people would close hoods more often when not in use. These two tactics, when used together, significantly reduced the number of times hoods were left open (as compared to regular behavior in a control building). A year later, only using the sticker worked well to maintain sustainable behavior. Using written communication is a low-cost strategy but the impact of using automated data as feedback for users is discussed as being very useful.

Another paper in this theme of encouraging behavioral change to improve energy use is titled "Energy Saving at Work: Exploring the Role of Social Norms, Perceived Control and Ascribed Responsibility in Different Office Layout." In it, Xu et al. utilize social and environmental psychology to re-iterate that reducing energy consumption in work settings-and office buildings in particular-is imperative to combat climate change. The team used the Theory of Planned Behavior (TPB) to understand psychological aspects of energy saving intentions and behaviors in the workplace. A sense of responsibility was found to be a strong predictor of this kind of intention for those working in single-occupancy offices, as well as shared offices. With respect to behavior, injunctive norms mattered for those in single-person offices; descriptive norms mattered for those in shared offices. The authors also found unexpected results related to perceived control, depending on office type-a result that will undoubtedly spark ideas for researchers in engineering and the social sciences.

The next two articles continue to center on work settings, particularly indoor environmental quality (IEQ). The paper titled "Reliability of an Item Set Assessing Indoor Climate in OfficesResults from Field Studies and Laboratory Research" explains that while robust results are reported concerning IEQ in the field, the reliability of questionnaire-based scales should be assessed often. Schakib-Ekbatan et al. do just that, both in the field and in the lab. Internal consistency for scales offered to participants in the lab environment were lower than those from the field, highlighting a need for researchers to consider psychometrics and context when interpreting self-reported data. 
Building on this result, the next paper is one that we, as Research Topic editors, submitted along with Kim et al., titled "Impact of office modernization on environmental satisfaction: A naturalistic field study." We merged objective measurements of lighting, acoustics, and indoor air quality with questionnaire data using scales and open-ended items to afford perceptual responses from occupants about their satisfaction with these attributes and with the level of input they had into a recent retrofit process. Satisfaction was higher after the retrofit; size of personal workspaces and a sense of privacy were especially important to employees, along with their sense of control. Because the level of input that occupants felt that they had correlated with their perceptions of environmental satisfaction after its completion, the paper supports the argument that involving occupants in design processes is beneficial for satisfaction and wellbeing at work.

The next paper, this time by Wells et al., concerns the role of nature in urban settings-but with an interesting twist. The paper is titled "Nearby Nature Buffers the Pain CatastrophizingPain Intensity Relation Among Urban Residents with Chronic Pain" and asks whether nearby amounts of nature, measured using satellite data, alters experiences of chronic pain among community-dwelling adults. Using diary data over 2 weeks, Wells et al. showed that nearby nature buffered the relation between catastrophizing and pain intensity. These results matter to public health practitioners and community planners seeking to improve quality of life and promote the benefits of nature in sustainable community models.

Finally, a submission by Alexander and Wydeman outlines a perspective about the ways in which environmental psychology blends with new urbanism (as well as the ways in which it does not). In their piece, "The Intersection and Divergence of New Urbanism and Environmental Psychology: An Exploration," a 1970s inner-city neighborhood in Vancouver, Canada is expressed as an example to distinguish between the two orientations. One take-away from the work is that planners, along with those who systematically examine human behavior, ought to read widely across disciplines to ensure that civic goals, the physical needs of residents, and the psychology of a community, can be met and remain relevant over time.

Many individuals around the world use environmental psychology to envision and develop healthy buildings and public infrastructure. Given the aims and results of the papers in this collection, it seems to us that social scientists are engaging with other practitioners in prudent ways. Deepening these relationships with inspiring and cogent findings in a collection like this may spur innovation-from technologies to capture, measure, and interpret human behavior, to precise psychological theories and methods to predict cognition and emotion for architectural attributes. Let's continue to work together to design for long-term satisfaction and pro-social and pre-environmental outcomes. We hope that this Research Topic moves its readers in exciting directions.

\section{AUTHOR CONTRIBUTIONS}

LM wrote the first draft of the editorial. AK edited it for clarity. All authors contributed to the article and approved the submitted version.

Conflict of Interest: The authors declare that the research was conducted in the absence of any commercial or financial relationships that could be construed as a potential conflict of interest.

Copyright $\odot 2020$ McCunn and Kim. This is an open-access article distributed under the terms of the Creative Commons Attribution License (CC BY). The use, distribution or reproduction in other forums is permitted, provided the original author(s) and the copyright owner(s) are credited and that the original publication in this journal is cited, in accordance with accepted academic practice. No use, distribution or reproduction is permitted which does not comply with these terms. 